\title{
ABORDAJE FARMACOTERAPÉUTICO DEL PACIENTE HOSPITALIZADO CON DESÓRDENES HIPERGLUCÉMICOS
}

\author{
Chaverri Fernández, José Miguel ${ }^{1}$; Cordero García, Eugenia ${ }^{1}$; Fallas Retana, Fallom ${ }^{3}$; \\ Montoya Calderón, Vivian ${ }^{3}$ y Zavaleta Monestel, Esteban ${ }^{2}$
}

1Profesor Departamento de Farmacología, Toxicología y Farmacodependencia de la Facultad de Farmacia de la Universidad de Costa Rica, San José, Costa Rica. ${ }^{2}$ Director de la Farmacia Hospital Clínica Bíblica, San José, Costa Rica. ${ }^{3}$ Estudiantes de Internado en Farmacia de la Universidad de Costa Rica, San José, Costa Rica.

Resumen: Justificación y Objetivo: Los desórdenes glucémicos están asociados a una mayor morbimortalidad en el ámbito hospitalario. El conocer la realidad actual y describir los modelos de abordaje farmacoterapéutico de los desórdenes glucémicos facilita la implementación de guías clínicas que promuevan el correcto uso de medicamentos.

Material y métodos: Se propuso con este fin realizar un estudio observacional retrospectivo, que incluyera a los pacientes hospitalizados en el Hospital Clínica Bíblica en el período de marzo - diciembre del 2012 con dicha problemática.

Resultados: Se recolectaron los datos de 159 pacientes ( $n=1692$ glucemias). Del total de hiperglucemias registradas, un 78\% no fueron tratadas con insulina de ningún tipo, 53 de las aplicaciones de insulina indicadas no se relacionaban con una hiperglucemia y en 38 ocasiones se utilizó una dosis diferente a la indicada por el médico tratante. En un 30\% de los internamientos los pacientes utilizaron hipoglucemiantes orales concomitantemente con insulina y en un $47 \%$ de los internamientos se utilizó dosis escalonada de insulina como parte del tratamiento.

Conclusión: El porcentaje de pacientes que se mantuvieron euglucémicos durante toda la estancia hospitalaria fue bajo ( $6 \%$ de los internamientos), se sugiere establecer mecanismos que faciliten la monitorización y aplicación de insulinas. La utilización conjunta de hipoglucemiantes orales e insulina puede llevar al paciente a presentar cuadros de hipoglucemias. Con base en los resultados obtenidos se sugiere elaborar una guía clínica que estandarice el manejo intrahospitalario de este tipo de desórdenes y armonizar la información existente en las bases de datos del hospital y el expediente electrónico del paciente. 
Palabras clave: Desórdenes glucémicos, Hiperglucemia, Insulinoterapia.

Recibido: 10 Enero 2014. Aceptado: 20 Marzo 2014. Publicado: 23 Abril 2014.

\title{
PHARMACOTHERAPEUTIC APPROACH OF INPATIENT HYPERGLYCEMIC DISORDERS
}

\begin{abstract}
Aim: Glycemic disorders are associated with an increased morbidity and mortality in hospitals. The knowledge of hospital's reality and the pharmacotherapeutic approach description facilitate the implementation of clinical guidelines that promote the proper use of these medications.

Material and methods: A retrospective observational study was done from March to December of 2012, and included patients hospitalized in the Clínica Bíblica Hospital, San José, Costa Rica.

Results: Data from 159 patients ( $\mathrm{n}=1692$ glycemic controls) was collected. Of all the hyperglycemic patients registered, $78 \%$ were not treated with any insulin, 53 of the insulin applications were not related to hyperglycemia, and in 38 occasions a different dose than the prescribed by the physician was used. In $30 \%$ of the cases patients used both oral hypoglycemic agents and insulin and in $47 \%$ of the times a sliding scale dose was used as part of treatment.

Conclusion: The percentage of patients who remained euglycemic throughout the hospital's stay was low (6\%); indicating that the establishment of mechanisms that facilitate the monitoring and implementation of insulin's dosage protocols are required. The combined use of oral hypoglycemic agents and insulin may lead the patient to hypoglycemia. Based on the results obtained, it is suggested that clinical guidelines must be elaborated in order to standardize hospital management of such disorders. Harmonization of existing information in hospital databases and electronic patient record is needed.
\end{abstract}

Key words: Glycemic disorders, Hyperglycemia, Insulin therapy

\section{INTRODUCCIÓN}

La hiperglucemia y la hipoglucemia en el ámbito hospitalario están asociadas con una mayor morbilidad y mortalidad en los pacientes [1-5], presentándose tanto en pacientes diabéticos como no diabéticos. La Asociación Americana de Diabetes (ADA, por sus siglas en inglés) publica en el año 2013 la guía más reciente sobre manejo de estos pacientes [2], la cual incluye los rangos de glucemias meta en pacientes hospitalizados. En el caso de los pacientes críticos tratados con insulina se busca mantener un rango inferior a $180 \mathrm{mg} / \mathrm{dl}$, esperando así que las glucemias permanezcan entre 140-180 mg/dl. Para pacientes no críticos, la glucemia preprandial debe ser menor a $140 \mathrm{mg} / \mathrm{dl}$ y la glucemia al azar menor de $180 \mathrm{mg} / \mathrm{dl}$. En pacientes no diabéticos se aceptan valores de glucemia en ayuno no mayores de $126 \mathrm{mg} / \mathrm{dl}$ y al azar no mayores de $200 \mathrm{mg} / \mathrm{dl}[1,6,7]$.

El manejo de los hospitalizados se basa en la utilización de infusiones continuas de insulina de acción rápida y la administración de un bolo de la misma insulina a una dosis que cubra la ingesta nutricional cuando se requiera. En algunos casos este método puede transferirse al régimen de insulina subcutáneo bolo-basal-correctivo, 
administrando entre el 60 y $80 \%$ de insulina total en forma de insulina basal y el restante en bolos que cubran los requerimientos nutricionales $[6,8]$.

De acuerdo con las guías clínicas recientes para pacientes no críticos, el régimen más fisiológico se basa en la aplicación de una insulina de acción lenta una vez al día o una de acción intermedia aplicada dos veces al día, esto en combinación con insulina de acción rápida en bolos preprandiales y con correcciones de dosis según la ingesta nutricional del paciente $[6,9]$.

Otro método utilizado es el esquema denominado en inglés "sliding scale" o dosis escalonada en el cual se administra insulina de acción corta dependiendo de la glucemia en un momento dado, sin tomar en cuenta los tiempos de comida, o la presencia o ausencia de otros regímenes de insulina. Es importante mencionar que dicho esquema se encuentra en desuso, ya que no cubre las necesidades basales de insulina y su utilización ha generado múltiples hipoglucemias e hiperglucemias de rebote, así como un pobre control glucémico $[2,10,11]$.

Ejemplo de esto son los resultados del estudio Rabbit 2, el cual comparó la eficacia y seguridad del régimen bolo-basal versus el "sliding scale" en pacientes diabéticos tipo 2; dicho estudio demostró que con el régimen bolo-basal se observa una mejora significativa en el control glucémico y reducción de complicaciones, sin aumentar el número de hiperglucemias e hipoglucemias severas [12].

Dentro de los medicamentos de uso crónico en los pacientes diabéticos se encuentran los distintos hipoglucemiantes orales. Los consensos recientes recomiendan evitar su uso durante la hospitalización debido a su falta de flexibilidad, su lento inicio de acción terapéutica, incidencia de hipoglucemias severas y sus potenciales efectos adversos. Por lo tanto se recomienda que al momento de admisión hospitalaria se suspendan estos medicamentos y se inicie insulinoterapia, a excepción de casos en que el paciente esté estable, pueda comer regularmente $y$ no tenga contraindicado el uso de los mismos $[2,8,13]$.
Una de las complicaciones más frecuentes de los pacientes a nivel hospitalario son las hipoglucemias; esta se define como una glucemia menor de $70 \mathrm{mg} / \mathrm{dl}$ y se considera severa cuando se presenta una glucemia menor de $40 \mathrm{mg} / \mathrm{dl}$, con un nivel de afectación cognitivo, en el que se requiere asistencia médica (administración de carbohidratos o glucosa por ejemplo). Es importante que para evitar una hipoglucemia la terapia antidiabética del paciente hospitalizado se evalúe cuando los valores sean iguales o menores a $100 \mathrm{mg} / \mathrm{dl}$ (mayores a $70 \mathrm{mg} / \mathrm{dl}$ ) y se modifique cuando los valores de glucemia sean menores $o$ iguales a $70 \mathrm{mg} / \mathrm{dl}[6,14]$.

El tratamiento de una hipoglucemia puede basarse en la utilización de carbohidratos orales y en casos severos se requiere la administración de dextrosa $50 \%$ por infusión intravenosa. Luego del tratamiento del episodio hipoglucémico la monitorización de la glucemia debe continuar hasta estabilizarse en niveles superiores a 80 $\mathrm{mg} / \mathrm{dl}[7,13]$.

El Hospital Clínica Bíblica es un hospital privado de aproximadamente 100 camas de capacidad, ubicado en San José, Costa Rica. Gracias al proceso de acreditación al que voluntariamente se somete el hospital por Joint Commission International (JCI) es que se buscan constantemente elaborar planes de mejora $\mathrm{y}$ establecer protocolos para el correcto uso o manejo de los medicamentos del paciente hospitalizado.

Considerando la importancia que tiene el manejo de los desórdenes hiperglucémicos y las consecuencias que un mal tratamiento trae es que se plantea realizar un análisis farmacoterapéutico del paciente hospitalizado con desórdenes hiperglucémicos tratados con insulinas, indagando el uso de las mismas de manera individual y las hipoglucemias asociadas presentadas durante su estadía en el centro hospitalario. Lo anterior busca facilitar la implementación de guías clínicas aprobadas por JCI que promuevan el correcto uso en el ámbito hospitalario de estos medicamentos a 
partir del conocimiento de la realidad actual y la descripción de los modelos de abordaje.

El período de estudio corresponde al tiempo establecido en el Plan de Mejora de Manejo de Pacientes con Desórdenes Glucémicos a desarrollarse en el Hospital Clínica Bíblica.

\section{MATERIALES Y MÉTODOS}

Con el fin de poder responder a los objetivos planteados en la investigación se propuso la realización de un estudio observacional de tipo retrospectivo, que abarcó a los pacientes hospitalizados en el Hospital Clínica Bíblica en el período del 1 marzo del 2012 al 31 de diciembre del 2012 y que durante su estadía les fuera prescrita insulina [15]. Se incluyeron en el estudio pacientes con internamientos mayores a los dos días y con una prescripción y administración de al menos una dosis de insulina. Los pacientes incluidos en el estudio se clasificaron en diabéticos y no diabéticos. Además, si la estadía del paciente fue en la unidad de cuidados intensivos, se clasificó como crítico. Se consideró como no crítico al paciente que fue admitido en otra área del hospital.

Para cada paciente se recopiló el valor de la glucemia por determinación con glucómetro (Optium Exceed Abbott), así como los reportados mediante exámenes de laboratorio. Se analizó si se encontraba euglucémico, hipoglucémico y/o hiperglucémico durante su estancia hospitalaria, conforme lo establecido en los Estándares de Cuidados Médicos en Diabetes, publicados en enero 2013 por la Asociación Americana de Diabetes [1]. De acuerdo con esta clasificación, en el caso de pacientes diabéticos críticos con insulinoterapia la glucemia debe mantenerse entre 140 y $180 \mathrm{mg} / \mathrm{dl}$; y en pacientes no críticos, se recomienda una glucosa preprandial menor a 140 $\mathrm{ml} / \mathrm{dl}$ y una glucemia tomada al azar menor a 180 $\mathrm{mg} / \mathrm{dl}$. En el caso de pacientes no diabéticos, la hiperglucemia hospitalaria se define como la glucemia en ayunas mayor o igual a $126 \mathrm{mg} / \mathrm{dl}$ y la glucemia al azar mayor o igual a $200 \mathrm{mg} / \mathrm{dl}$ [2,3,16-18].
Se define para nuestro análisis que un paciente se encuentra hiperglucémico si su glucemia se encontró mayor a $180 \mathrm{mg} / \mathrm{dl}$, esto incluye al paciente diabético crítico, no crítico o no diabético y se consideró una hipoglucemia cuando las glucemias fueron menores de $70 \mathrm{mg} / \mathrm{dl}[13,18$ 20].

Para el análisis del manejo farmacológico de los desórdenes hiperglucémicos se determinó el abordaje de la hiperglucemia según la insulina administrada y el tiempo que se tardó en aplicar la misma al paciente. Para la recopilación de información se utilizó una hoja para la recolección de datos, en la cual se detallan las glucemias, insulinas aplicadas (Insulina Regular (Reg), Insulina Glargina (Glargina), Insulina NPH (NPH), Insulina Premezclada 70/30 (70/30), Insulina Lispro (Lispro), Infusión de Insulina Regular (Infusión R)), hipoglucemiantes utilizados en conjunto con la insulinoterapia y la aplicación de dextrosa al $50 \%$ en casos de hipoglucemias, entre otros datos; dicha información fue suministrada por el departamento de informática a través de las bases de datos del Hospital Clínica Bíblica, así como los expedientes físicos de los pacientes.

Para el desarrollo de este estudio se contó con la aprobación de la Dirección Médica y el Comité de Farmacoterapia del Hospital. Se garantizó el manejo adecuado y la confidencialidad de los datos de los pacientes incluidos en el estudio mediante sistemas de codificación que imposibilitan la identificación de los mismos.

\section{RESULTADOS}

Se recolectaron los datos de 159 pacientes, de los cuales el $36 \%$ fueron mujeres y $64 \%$ hombres, con edades entre los 16 y 95 años (72 años en promedio). De estos pacientes, el $82 \%$ tenían un diagnóstico establecido de diabetes mellitus, mientras que el restante $18 \%$ no eran diabéticos, pero utilizaron insulina durante su estancia hospitalaria.

Es importante destacar que algunos de los pacientes fueron internados más de una vez

Revista electrónica publicada por el Departamento de Farmacología de la Escuela de Medicina de la Universidad de Costa Rica, 2060 San José, Costa Rica. ${ }^{\circledR}$ All rights reserved. Licensed under a Creative Commons Unported License. 
durante el periodo en el cual se llevó a cabo este estudio, por lo que aún cuando el número de pacientes fue de 159, el número registrado de internamientos fue de 187. Los resultados del estudio están basados respecto al número de internamientos, esto debido a la posibilidad que se presentaran variaciones en las condiciones, lugar de internamiento y medicamentos utilizados. Un $92 \%$ de los internamientos analizados correspondió a pacientes no críticos (n=172). Del total de internamientos analizados, sólo un $6 \%$ [11] de los pacientes se mantuvieron euglucémicos durante toda su estancia. De estos pacientes euglucémicos ninguno estuvo internado en la Unidad de Cuidados Intensivos.

Se analizaron 1692 glucemias, de las cuales 96\% fueron tomadas mediante glucómetros y $4 \%$ fueron reportadas por el laboratorio clínico. Del total de glucemias reportadas, en un $96 \%$ de los internamientos se registraron hiperglucemias; los rangos oscilaron entre $181 \mathrm{mg} / \mathrm{dl}$ y $914 \mathrm{mg} / \mathrm{dl}$, con un promedio de $239 \mathrm{mg} / \mathrm{dl}$.

En las figuras 1, 2 y 3 se puede observar la proporción de insulinas utilizadas y sus posibles combinaciones.

En un $47 \%$ de los internamientos se utilizó "sliding scale" como parte del tratamiento de las hiperglucemias. En un 18\% de los casos no se aplicaron las unidades de insulina tal y como se indicaba en el esquema asignado por el médico tratante.

De la misma forma se encontró que en menos de un $1 \%$ de los internamientos que requerían manejo inmediato de hiperglucemias se utilizaron insulinas de larga acción o acción intermedia (insulina NPH y glargina). Del total de hiperglucemias registradas un $78 \%$ no fueron tratadas con insulina de ningún tipo.

Se realizaron 368 aplicaciones de insulinas de acción inmediata durante el período de estudio. En estos casos en promedio se tardó 25 minutos desde la detección de la hiperglicemia en el paciente hasta la aplicación de la insulina, mientras que en un 33\% de los casos no hubo retraso en la aplicación de la misma. Es importante mencionar que en 50 de los internamientos analizados la hora de aplicación de la insulina se reportaba antes de la toma de la glucemia (hiperglucemia en este caso); 53 de las aplicaciones de insulina indicadas inmediatamente no se relacionaban con una hiperglucemia y en 38 ocasiones se utilizó una dosis diferente a la indicada por el médico tratante.

En un 30\% de los internamientos los pacientes utilizaron hipoglucemiantes orales concomitantemente con el uso de insulinas, siendo estos: metformina, glimepiride, glibenclamida, glicazide, vildagliptina, sitagliptina y combinaciones de metformina con sitagliptina.

Con respecto a las hipoglucemias de los internamientos analizados, se reportaron 72 glucemias menores de $70 \mathrm{mg} / \mathrm{dl}$, lo cual corresponde a un $4 \%$ del total de glucemias del estudio, con rangos que oscilan entre $20 \mathrm{mg} / \mathrm{dl} \mathrm{y}$ $69 \mathrm{mg} / \mathrm{dl}$, con un promedio de $55 \mathrm{mg} / \mathrm{dl}$. De las 72 hipoglucemias reportadas se utilizó dextrosa $50 \%$ en un $13 \%$ de los casos y en promedio se tardó 26 minutos desde la detección de la hipoglucemia en el paciente hasta la aplicación de la dextrosa. En siete de las ocasiones en las que se utilizó dextrosa al $50 \%$ no se encontró una relación entre la utilización de la misma y una hipoglucemia reportada. Además, en 42 de las hipoglucemias reportadas no se utilizó dextrosa $50 \%$ como tratamiento.

\section{DISCUSIÓN}

Tomando en cuenta que se trabajó tanto con pacientes diabéticos como no diabéticos y con un amplio rango de edades se pudo realizar un análisis más global del tratamiento con insulinas en los pacientes internados en este centro hospitalario.

La dosificación del tratamiento no se pudo analizar en detalle, ya que sólo un $28 \%$ de los pacientes tenían reportado su peso en el expediente, lo cual no permitió realizar un cálculo exacto de la dosis necesaria de insulina tomando 
en cuenta el peso así como la condición particular de la persona.

El porcentaje de pacientes que se mantuvieron euglucémicos durante toda la estancia hospitalaria fue bajo (6\%), esto se puede deber tanto a errores en la farmacoterapia como a las condiciones patológicas del paciente, sabiendo que una situación de estrésfisiológico puede estar relacionada con una hipoglucemia o una hiperglucemia [1].

El uso de la insulina regular como monoterapia respondió a la necesidad de administración inmediata en momentos donde el paciente se encontraba en hiperglucemia y necesitaba alcanzar los valores meta. Sin embargo, el uso de insulina regular en bolo podría generar hipoglucemias o hiperglucemias de rebote, por

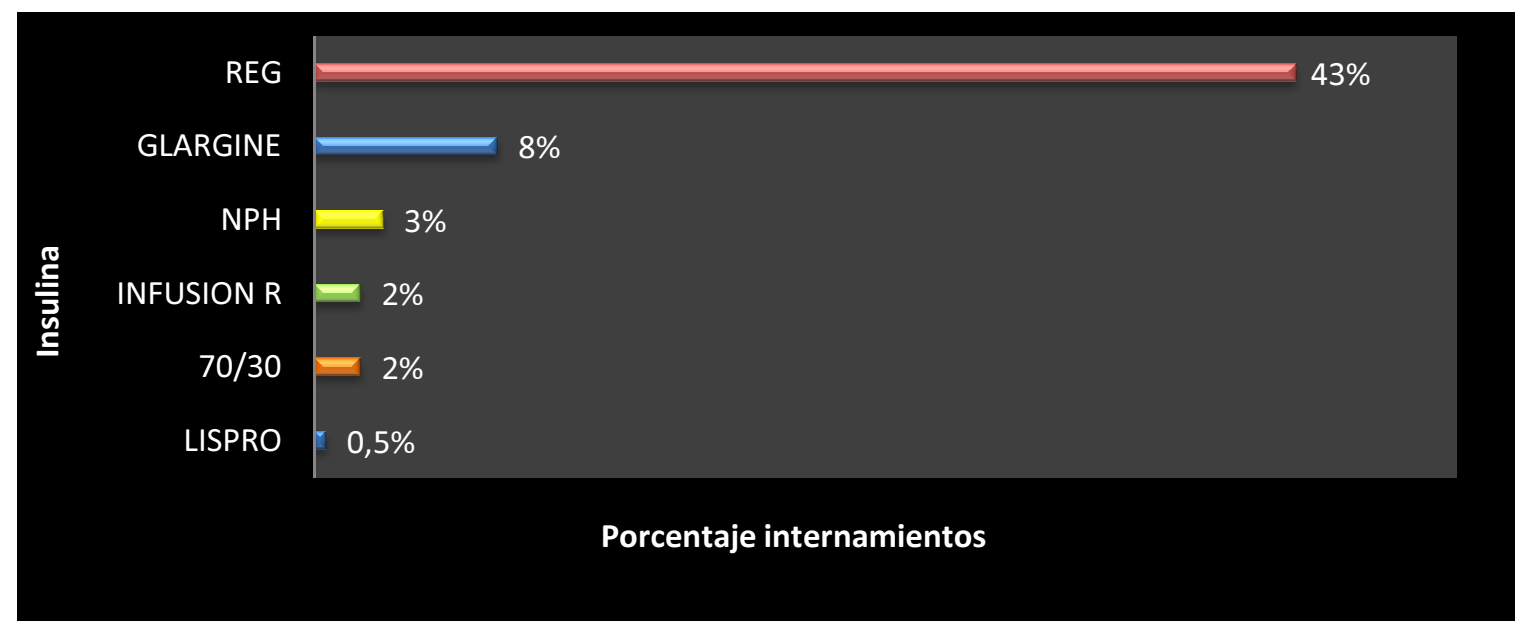

Figura 1. Distribución de insulinas utilizadas como monoterapia

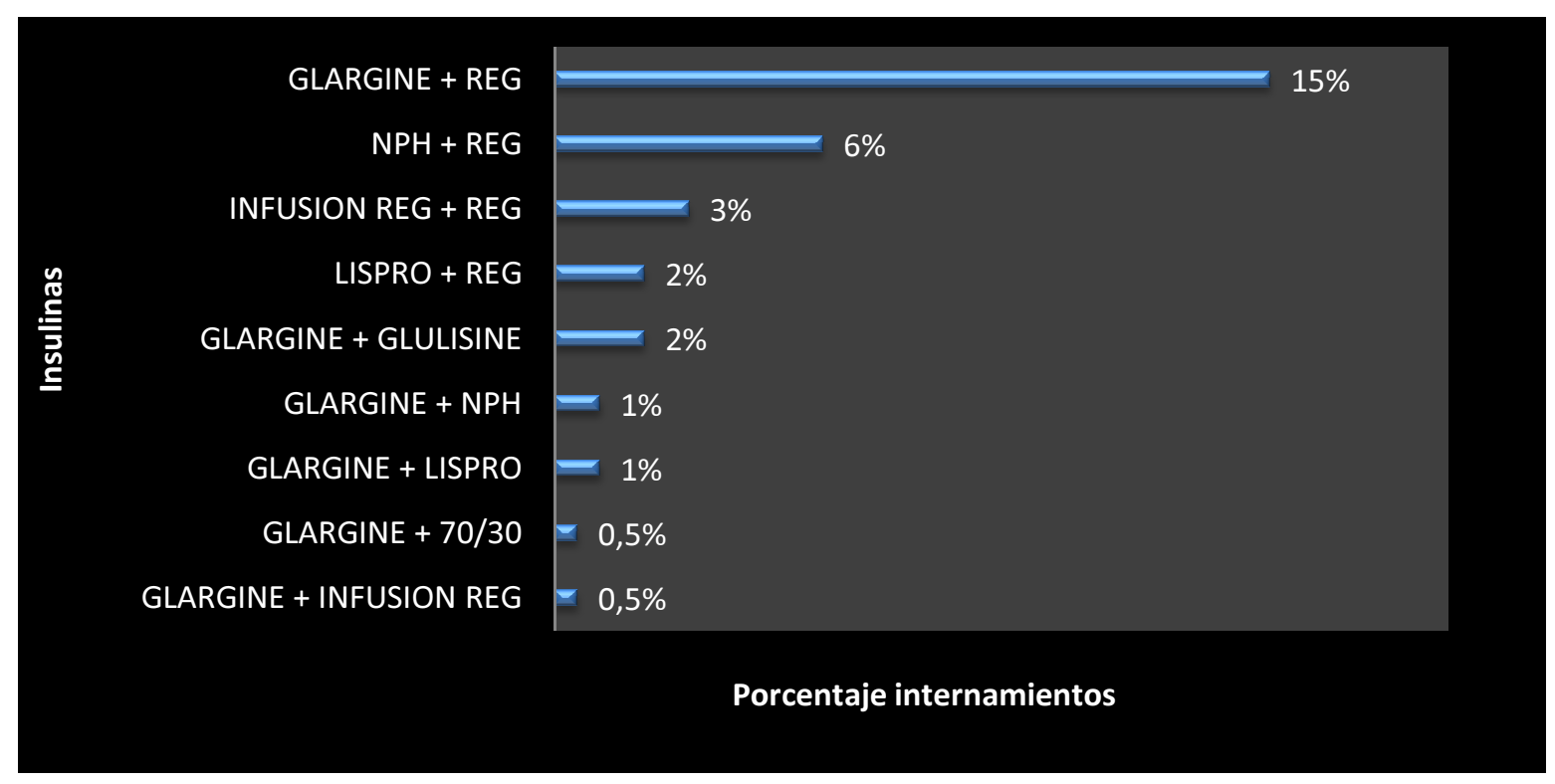

Figura 2. Distribución de insulinas utilizadas en combinación dual

Revista electrónica publicada por el Departamento de Farmacología de la Escuela de Medicina de la Universidad de Costa Rica, 2060 San José, Costa Rica. ${ }^{\circledR}$ All rights reserved. Licensed under a Creative Commons Unported License. 


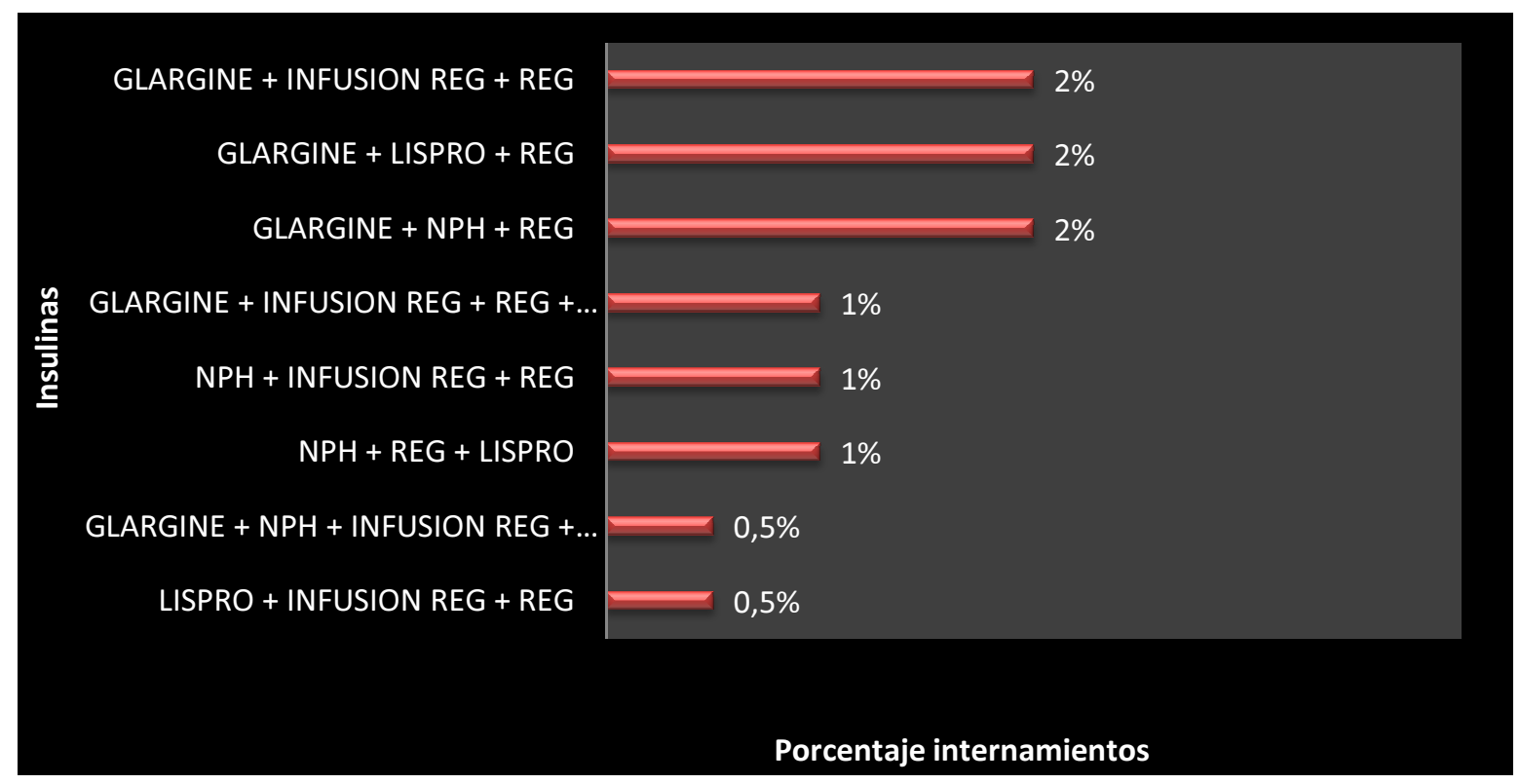

Figura 3. Distribución de insulinas utilizadas en combinaciones múltiples

lo que su uso como monoterapia es considerado controversial [10].

En el caso de la combinación de insulina regular e insulina glargina, este esquema suele responder a lo que se considera el régimen más fisiológico para el tratamiento de la hiperglucemia según las guías de manejo intrahospitalario más actuales [9]. Las demás combinaciones se utilizaron en una medida relativamente similar pero menos frecuente, por lo que el abordaje utilizado se acerca, más no completamente, a lo recomendado por las guías internacionales de manejo de hiperglucemias [11,12].

El régimen de "sliding scale" fue ampliamente utilizado, aunque cabe destacar que la aplicación no fue correcta en un $18 \%$ de los casos, lo cual es preocupante ya que si este no se sigue adecuadamente podría llevar potencialmente a un descontrol glucémico innecesario [4].

Lo mismo sucede en los casos en que se utilizó de forma inmediata insulina de acción intermedia o lenta para tratar una hiperglucemia, ya que dichos tipos de insulina se deben usar para mantener los niveles basales de glucosa en sangre y no para tratar los picos de hiperglucemia presentes de forma espontánea en un paciente, siendo así una terapia poco efectiva.

Otra recomendación fundamental es que la aplicación de estas insulinas debe darse en el momento preciso en que el paciente lo requiere y, tal como se observó, en un $67 \%$ de los casos se dio un retraso en la aplicación de la misma. Lo anterior se pudo deber a múltiples factores, tales como situaciones que ameritan retrasar la aplicación por parte del personal de enfermería, una mayor duración en la localización del médico tratante, retraso en el envío del medicamento desde la farmacia, fallos en el sistema de registro de la aplicación de la insulina, entre otros.

En un $78 \%$ de las hiperglucemias no se utilizó ningún tipo de insulina y se dieron casos en que la aplicación no se asociaba a una hiperglucemia o la aplicación se realizó en una dosis diferente a la indicada por el médico; lo anterior deja claro que eventualmente la aplicación de un protocolo de manejo de desórdenes

Revista electrónica publicada por el Departamento de Farmacología de la Escuela de Medicina de la Universidad de Costa Rica, 2060 San José, Costa Rica. ${ }^{\circledR}$ All rights reserved. Licensed under a Creative Commons Unported License. 
glucémicos y el seguimiento por parte de un farmacéutico clínico pueden ser de gran beneficio para evitar este tipo de situaciones, que representan un riesgo para el paciente.

Es importante que se tome en cuenta que la utilización conjunta de hipoglucemiantes orales e insulina puede llevar al paciente a presentar cuadros de hipoglucemias, así como potenciales efectos adversos, por lo cual es recomendable monitorizar estos pacientes, vigilar su estado durante el internamiento y en caso necesario suspender dichos medicamentos según lo indiquen las recomendaciones internacionales $[4,8]$.

Debido a que el tiempo de retraso en la aplicación tanto de insulina como de la dextrosa $50 \%$ es similar, se podría pensar que los factores de retraso podrían ser los mismos en ambos casos. No es posible determinar la razón por la cual se obtuvo un bajo porcentaje de uso de dextrosa $50 \%$, debido a que no se conocía la condición del paciente en el momento que presentó la hipoglucemia o si esta fue manejada mediante otras terapias.

Es muy importante que las autoridades hospitalarias evalúen armonizar los valores existentes suministrados en las bases de datos del hospital y el expediente electrónico del paciente, con el fin de eliminar las divergencias entre ellos. Para un análisis más amplio de la insulinoterapia se debería contar con el peso de los pacientes, así como la condición del mismo, para poder calcular si las dosis utilizadas fueron apropiadas individualizando cada caso clínico (0.3-1.2 U/Kg/día según la situación específica).

Se recomienda la realización de una futura investigación que analice cuáles son los factores

\section{REFERENCIAS}

1. Umpierrez GE, Hellman R, Korytkowski MT, Kosiborod M, Maynard G, Montori V, et al. Management of Hyperglycemia in Hospitalized Patients in Non-Critical Care Setting: An Endocrine determinantes que llevan a errores en la aplicación de la insulinoterapia y los factores que generan errores en el manejo del paciente con insulinas, por lo que se sugiere implementar una guía clínica que estandarice el manejo intrahospitalario de este tipo de pacientes.

\section{CONCLUSIONES}

Es muy importante que las autoridades hospitalarias evalúen armonizar los valores existentes suministrados en las bases de datos del hospital y el expediente electrónico del paciente.

Para un análisis más amplio de la insulinoterapia se debería contar con el peso de los pacientes, así como la condición del mismo, para poder calcular si las dosis utilizadas fueron apropiadas individualizando cada caso clínico (0.3$1.2 \mathrm{U} / \mathrm{Kg} /$ día según la situación específica).

El porcentaje de pacientes que se mantuvieron euglucémicos durante toda la estancia hospitalaria fue bajo, se sugiere establecer mecanismos que faciliten la monitorización y aplicación de insulinas.

Es necesario la implementación de una guía clínica que estandarice el manejo intrahospitalario de este tipo de pacientes y minimice cualquier riesgo asociado a su manejo intrahospitalario. Se recomienda la realización de una futura investigación que analice cuáles son los factores determinantes que llevan a errores en la aplicación de la insulinoterapia y los factores que generan errores en el manejo del paciente con insulinas.

Society Clinical Practice Guideline. Journal of Clinical Endocrinology \& Metabolism. 2012; 97(1):16-38.

2. American Diabetes Association. Standards of medical care in diabetes-2013. Diabetes care [Internet]. 2013; 36(1):11-66. Disponible en: http://www.ncbi.nlm.nih.gov/pubmed/23264422.

3. Moghissi E, Donahue M, Ismail-Beigi F, Korytkowski M, McDonnell M. Revisiting Inpatient Hyperglycemia: New Recommendations, Evolving Data, and Practical

Revista electrónica publicada por el Departamento de Farmacología de la Escuela de Medicina de la Universidad de Costa Rica, 2060 San José, Costa Rica. ${ }^{\circledR}$ All rights reserved. Licensed under a Creative Commons Unported License. 
Implications for Implementation. Postgraduate Institute for Medicine, Global Directions in Medicine, Inc. \& Novo Nordisk Inc. 2009: 3-30.

4. Botella M, Rubio JA, Percovich JC, et al. Glycemic control in non-critical hospitalized patients. Endocrinologia y Nutrición. 2011; 58(10):536-40.

5. Kitabchi A, Freire A, Umpierrez G. Evidence for strict inpatient blood glucose control: time to revise glycemic goals in hospitalized patients. Metabolism Clinical and Experimental. 2008; 57: 116 -20.

6. Krinsley J. Glycemic Control, Diabetic Status, and Mortality in a Heterogeneous Population of Critically Ill Patients before and During the Era of Intensive Glycemic Management: Six and One-Half Years Experience at a University-Affiliated Community Hospital. Thoracic and Cardiovascular Surgery. 2006: 317-25.

7. Klonoff DC, Buckingham B, Christiansen J, Montori VM, et al. Continuous Glucose Monitoring: An Endocrine Society Clinical Practice Guideline. The Endocrine Society's Clinical Guidelines. Journal of Clinical Endocrinology and Metabolism. 2011; 96 (10): 2968-79.

8. Wesorick D, O’Malley C, Rushakoff R, Larsen K, Magee M. Management of Diabetes and Hyperglycemia in the Hospital: A Practical Guide to Subcutaneous Insulin Use in the Non-Critically Ill, Adult Patient. Journal of Hospital Medicine [Internet]. 2008 Sep [cited 2013 May 24]; 3(5):17$28 . \quad$ Available from: http://www.ncbi.nlm.nih.gov/pubmed/18951381.

9. Barnard K, Batch BC, Lien LF. Subcutaneous Insulin: A Guide for Dosing Regimens in the Hospital. Glycemic Control in the Hospitalized Patient. A Comprehensive Clinical Guide. 2011. p. 7-16.

10. Cheung NW, Chipps DR. Sliding scale insulin: will the false idol finally fall? Internal Medicine Journal [Internet]. 2010 Sep 15 [cited 2013 Jun 1]; 40(9):662-4. Available from: http://doi.wiley.com/10.1111/j.14455994.2010.02231.x.

11. Fiore LD, Brophy M, Ferguson RE, D'Avolio L, Hermos J a, Lew $\mathrm{R}$ a, et al. A point-of-care clinical trial comparing insulin administered using a sliding scale versus a weight-based regimen. Clinical trials [Internet]. 2011 Apr [citado el 24 mayo 2013]; $8(2): 183-95$. Disponible en:http://www.pubmedcentral.nih.gov/articlerende r.fcgi?artid=3195898\&tool=pmcentrez\&rendertype= abstract.
12. Umpierrez GE, Smiley D, Zisman A, Prieto L, Palacio A, Ceron M, et al. Randomized Study of Basal-Bolus Insulin Therapy in the Inpatient Management of Patients with Type 2 Diabetes (RABBIT 2 Trial). Diabetes Care. 2007; 30(9):2181-6.

13. Tomky D. Detection, Prevention, and Treatment of Hypoglycemia in the Hospital. Diabetes Spectrum. 2005; 18(1):39-44.

14. American Diabetes Association Workgroup on Hypoglycemia. Defining and Reporting Hypoglycemia in Diabetes. Diabetes Care. 2005; 28(5):1245-9.

15. Departamento de Farmacia. Plan de Mejora de Manejo de Pacientes con Desordenes Glucémicos. Hospital Clínica Biblica. 2013.

16. Schmeltz L. Management of Inpatient Hyperglycemia. Laboratory Medicine [Internet]. 2011;42(7):427-34. Disponible en: http://labmed.ascpjournals.org/cgi/doi/10.1309/L MU72V7QWUDUYQIJ.

17. Moghissi ES, Korytkowski MT, DiNardo M, Einhorn D, Hellman R, Hirsch IB, et al. American Association of Clinical Endocrinologists and American Diabetes Association Consensus Statement on Inpatient Glycemic Control. Endocrine Practice [Internet]. 2009;15(4):353-69. Disponible en: http://www.ncbi.nlm.nih.gov/pubmed/19454396.

18. Ugaz Zegarra M. Reformulación de consensos posNICE-SUGAR en el manejo de hiperglicemia intrahospitalaria para el médico internista. Revista del Cuerpo Médico Hospital Nacional Almanzor Aguinaga Asenjo [Internet]. Cuerpo Médico del Hospital Nacional Almanzor Aguinaga Asenjo; 2011; 4(1):45-52. Disponible en:

http://dialnet.unirioja.es/servlet/articulo?codigo=4 060190\&info=resumen\&idioma=SPA.

19. Braithwaite SS, Buie MM, Thompson CL, Baldwin DF, Oertel MD, Robertson BA, et al. ACE Inpatient Diabetes and Metabolic Control Consensus Conference: Hospital Hypoglycemia: Not Only Treatment but also Prevention. Endocrine. 2004;10(April):89-99.

20. American Diabetes Association Workgroup on Hypoglycemia. Defining and Reporting Hypoglycemia in Diabetes. Diabetes Care. 2005; $28(5)$. 


\section{INFORMACION DE AUTOR:}

Chaverri, José Miguel. Departamento de Farmacología, Toxicología y Farmacodependencia de la Facultad de Farmacia. Universidad de Costa Rica.

Email jose.chaverri@ucr.ac.cr Tel +506 2511-8329 